Disclosure of Interest: None declared

DOI: 10.1136/annrheumdis-2017-eular.6337

\section{FRI0309 CARDIOVASCULAR EVENTS IN ANCA-ASSOCIATED VASCULITIS: A META-ANALYSIS OF OBSERVATIONAL STUDIES}

E. Houben ${ }^{1}$, E.L. Penne ${ }^{1}$, A.E. Voskuyl ${ }^{2}$, J.W. van der Heijden ${ }^{3}$, M. Boers ${ }^{2,4}$ T. Hoekstra ${ }^{3} .{ }^{1}$ Internal Medicine, Northwest Clinics, Alkmaar; ${ }^{2}$ Amsterdam Rheumatology and Immunology Centre; ${ }^{3}$ Nephrology; ${ }^{4}$ Epidemiology and Biostatistics, VU University Medical Centre, Amsterdam, Netherlands

Background: Several chronic inflammatory diseases are associated with cardiovascular disease, but the cardiovascular risk in ANCA-associated vasculitis is poorly quantified.

Objectives: The aim of the present study is to review the evidence for the increased cardiovascular risk in patients with ANCA-associated vasculitis.

Methods: A comprehensive systematic review was conducted in accordance with guidelines of preferred reporting items for systematic reviews and meta-analyses (PRISMA). The databases PubMed, Embase.com and the Cochrane Library (Wiley) were searched for original observational studies reporting an estimate of the association between ANCA-associated vasculitis and cardiovascular events, including ischemic heart disease, cerebrovascular accidents and/or peripheral arterial disease. The quality of the included studies was assessed with the Newcastle-Ottawa Scale. Summary estimates were derived with a randomeffects model and reported as relative risks.

Results: 1375 studies were identified and 7 studies were included comprising 14098 ANCA-associated vasculitis patients versus general population controls in 6 studies and chronic kidney disease patients in 1 study. ANCA-associated vasculitis carried a relative risk of 1.65 (95\% confidence interval, 1.23-2.22) for all cardiovascular events, 1.60 (1.39-1.84) for ischemic heart disease and 1.20 $(0.98-1.48)$ for cerebrovascular accidents. We did not find studies that addressed the risk for peripheral arterial disease separately. No heterogeneity was seen in the estimates.

Conclusions: This meta-analysis of observational studies supports an increase in cardiovascular risk of about $65 \%$ in patients with ANCA-associated vasculitis, similar to that found in other chronic inflammatory diseases. Hence, there is a clear need for active cardiovascular risk management in patients with ANCA-associated vasculitis.

Disclosure of Interest: None declared

DOI: 10.1136/annrheumdis-2017-eular.1617

\section{FRI0310 LONG-TERM MORTALITY AND COMPLICATIONS IN YOUNG AND ELDERLY PATIENTS WITH ANCA-ASSOCIATED VASCULITIS}

A. Berti ${ }^{1}$, M. Felicetti ${ }^{2}$, R. Padoan ${ }^{2}$, G. Brunori ${ }^{3}$, F. Schiavon ${ }^{2}$, G. Paolazzi ${ }^{1}$ ${ }^{1}$ Rheumatology Unit, S.Chiara Hospital, Trento; ${ }^{2}$ Rheumatology Unit, Department of Medicine DIMED, Padua; ${ }^{3}$ Nephrology Unit, S. Chiara Hospital, Trento, Italy

Background: Advancing age is a risk factor for complications and mortality in anti-neutrophil cytoplasmic antibody (ANCA)-associated vasculitis (AAV)..$^{1,2}$

Objectives: To analyze differences in infectious, metabolic and cardiovascular complications, renal function, and mortality in patients diagnosed with AAV before or after 65 years of age, and followed for up to 5 years.

Methods: We retrospectively collected long-term clinical and laboratory data of AAV patients of two referral centers in Northern Italy from 2000, and grouped patients in young ( $\mathrm{YP},<65$ years old) and elderly (EP, $>65$ years old).

Results: Of the 114 patients included, 83 had a follow-up of at least 2 years (58 YP vs 25 EP). Median follow-up was 55 [32-100] months in YP and 44 [18-59] months in EP ( $\mathrm{p}<0.013) .86 .1 \%$ (68 patients) and $62.9 \%$ (22 patients) were diagnosed with GPA in YP and EP subsets, respectively. At baseline, YP and EP patients were similar in terms of BVAS/WG score, glomerulonephritis and alveolar hemorrhage, whereas creatinine levels $(1.1 \mathrm{mg} / \mathrm{dL}$ [0.8-2.3] vs 1.82 $\mathrm{mg} / \mathrm{dL}$ [0.98-3.5], $\mathrm{p}=0.044)$, renal insufficiency rate $(44.7 \%$ vs $67.6 \%, p=0.026)$ and ANCA pattern (PR3 $67.5 \%$ vs $26.5 \%$, MPO $19.5 \%$ vs $59.8 \% ; p<0.001$ for both comparisons) were different in $Y R$ and ER, respectively. No significant difference in induction and maintenance regimens was found in the two groups, nor in clinical remission rate after induction treatment (all $p>0.05$ ).

At 2 year, creatinine levels (YP $1.0 \mathrm{mg} / \mathrm{dL}$ [0.85-1.31], EP $1.2 \mathrm{mg} / \mathrm{dL}$ [1.1-2.2]) and renal insufficiency rate decreased within each group $(p<0.05)$. End-stage renal disease, hypertension rate, cardiac or cerebral ischemic attack rate, diabetes, solid or hematological cancer rate and mean vasculitis damage index were not statistically different in the two groups, whereas heart failure was more represented in EP $(0.0 \%$ vs $8.3 \%, p=0.027)$.

Within the first 5 years of follow-up, severe infection (requiring hospitalization) and mortality rates were significantly higher in EP group when compared with YP group $(p=0.024$ and $p=0.010$ by Kaplan-Meier analysis, respectively, Figure $1)$, mirrored by a higher annual severe infection rate $(p=0.041 ; 0.22 \pm 0.69$ versus $0.05 \pm 0.17)$ and annual mortality rate $(p=0.001 ; 0.33 \pm 1.02$ versus $0.01 \pm 0.08)$ in EP group. Relapse rate was similar in YP and EP within 5 years (Figure 1). Lymphopenia rate (at least 1 event, $<1000 \times 10^{9} / \mathrm{L}$ ) was significantly higher in EP only at 6 month $(p<0.05)$, whereas severe lymphopenia $\left(<500 \times 10^{9} / L\right)$, leukopenia
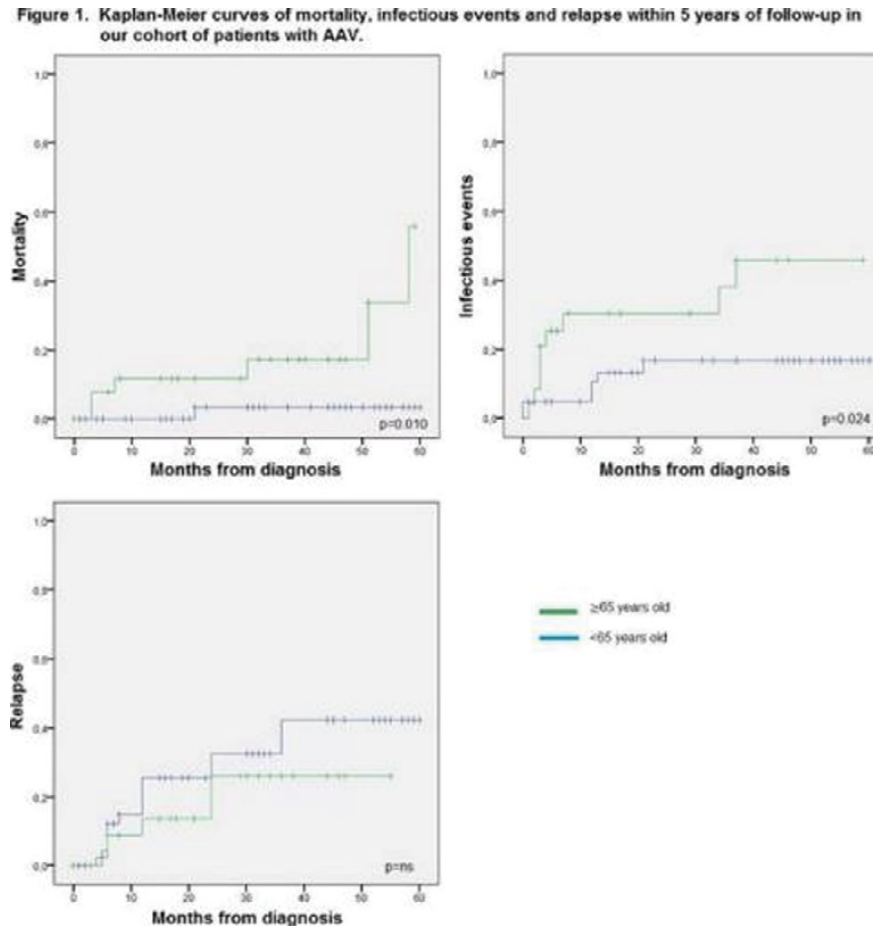

$\left(<4000 \times 10^{9} / \mathrm{L}\right)$ or hypogammaglobinemia $(\mathrm{lg}<5 \mathrm{~g} / \mathrm{L})$ rates were similar in both groups during the follow-up. Persistent lymphopenia ( $\geq 12$ months, $<1000 \times 10^{9} / \mathrm{L}$ ) was detected in 3 patients after cyclophosphamide treatment ( 2 YP and 1 EP). Only relapse before 2 years of follow-up was associated with infections in YP $(p<0.001$, OR 4.0 [Cl 95\%, 1.2-13.3]), but not in EP.

Conclusions: Heart failure is more frequent in older patients, which have higher infection and mortality rates. Transient lymphopenia is significantly higher in EP after induction treatment, but is not associated with their increase in infectious events. Despite a similar incidence of relapse in YP and EP, relapsing disease associates with infectious events in YP, but not in EP.

References:

[1] Flossmann O et al. Ann Rheum Dis. 2011 Mar;70(3):488-94.

[2] Timlin $\mathrm{H}$ et al. Semin Arthritis Rheum. 2015 Aug;45(1):67-9.

Disclosure of Interest: None declared

DOI: 10.1136/annrheumdis-2017-eular.5595

\section{FRI0311 ENDOVASCULAR INTERVENTION VERSUS SURGERY IN PATIENTS WITH TAKAYASU ARTERITIS: A META-ANALYSIS}

J.H. Jung, G.G. Song, S.J. Choi, Y.H. Lee, J.-H. Kim. Internal Medicine, Korea University Medical Center, Seoul, Korea, Republic Of

Background: Although medical treatment has advanced, surgical treatment is needed to control the progression and symptoms of Takayau arteritis (TA). Endovascular intervention or surgical revascularization is performed; however, there are few comparative studies of these methods.

Objectives: There are many studies about surgery and endovascular intervention; however, it is still unclear which treatment has better a benefit/risk ratio. Because neither meta-analysis nor large-scale studies are available for surgical treatment of TA, we conducted a meta-analysis to examine the outcome of surgical treatment. Methods: A meta-analysis comparing endovascular intervention and surgery outcomes was performed using the MEDLINE and Embase databases.

Results: A total of 14 studies of 598 patients and 1,049 lesions were included. Endovascular intervention was performed in 418 lesions and surgery in 631 lesions. Restenosis was more common in endovascular intervention than in surgery (odds ratio $[\mathrm{OR}]=2.74,95 \%$ confidence interval $[\mathrm{Cl}]=1.75-4.27, p<0.00001$ ). Other complications, including stroke, did not differ between endovascular intervention and surgery $(\mathrm{OR}=0.75,95 \% \mathrm{Cl}=0.49-1.15, p=0.19)$. There was no difference in mortality between the two groups $(\mathrm{OR}=1.11,95 \% \mathrm{Cl}=0.50-2.46, p=0.81$ ).

Table 1. Outcomes of endovascular intervetion compared to sugery in patients with Takayasu artheritis

\begin{tabular}{lccccccc}
\hline & \multicolumn{3}{c}{ Test of association } & & \multicolumn{3}{c}{ Test of heterogeneity } \\
\cline { 2 - 4 } \cline { 7 - 8 } & OR & $95 \% \mathrm{Cl}$ & P-value & & Model & P-value & $\mathrm{I}^{2}(\%)$ \\
\hline Restenosis & 2.74 & $1.75-4.27$ & $<0.00001$ & & $\mathrm{R}$ & 0.05 & 41 \\
Other complications & 0.75 & $0.49-1.15$ & 0.19 & & $\mathrm{~F}$ & 0.27 & 18 \\
Stroke & 0.5 & $0.17-1.50$ & 0.22 & & $\mathrm{~F}$ & 0.98 & 0 \\
Death & 1.11 & $0.50-2.46$ & 0.81 & & $\mathrm{~F}$ & 0.67 & 0 \\
\hline
\end{tabular}

Conclusions: This meta-analysis showed the superiority of surgical revascularization over endovascular intervention. 